\title{
Increasing the Stability and Quality of High-resolution FIB-SEM Tomography
}

Tobias Volkenandt, Fabián Pérez Willard and Benjamin Tordoff

Carl Zeiss Microscopy GmbH, Oberkochen, Baden-Wurttemberg, Germany

Focused ion beam scanning electron microscopy (FIB-SEM) tomography enables high-resolution, sitespecific characterization in 3D. In an iterative process, the ion beam removes a slice of material before an SEM image is acquired, as illustrated in Figure 1. This way a data stack is generated slice by slice that can be used afterwards to reconstruct the information about the volume of interest. Depending on the voxel size, volumes of up to several tens of microns in each direction can be acquired in manageable time. Using advanced slice tracking techniques (i.e. True- $Z$ functionality), it is possible to achieve an isotropic voxel resolution of below $5 \mathrm{~nm}$ [1]. Since the process is destructive, an important factor is also to capture as much information from the sample as possible. Therefore, the workflow is not only restricted to SEM imaging but can be combined with analytic mapping methods like energy dispersive X-ray spectroscopy (EDS) or electron backscatter diffraction (EBSD). Most recently, the latter is also available in the socalled static geometry, which turns any stage movement during the acquisition unnecessary. This of course reduces the acquisition time and increases the stability which leads to higher quality of the gathered data.

However, even during a solely imaging tomography, instabilities can occur stemming from external sources or from the sample itself. Examples for external influences are e.g. acoustic or electromagnetic vibrations. Those can be mitigated by better shielding and damping. Sample-induced instabilities occur when the sample is charging or degrades under the influence of the beams. To avoid charging, samples are often sputter coated with a thin metal layer prior to the investigation. However, if this is not possible or not sufficient to assure a homogeneous acquisition, another solution is to reduce the doses of the respective beams. Unfortunately, this means less material removal by FIB and less signal to noise during SEM imaging, which until now rendered the whole workflow impractical. In this contribution we will present an improved FIB-SEM tomography workflow especially for challenging samples, called Thin \& Fast tomography. It allows to remove thinner slices of material by FIB and image at faster speeds with the SEM without sacrificing image quality. Thus, a more stable run and subsequently more consistent image data can be achieved.

Besides explaining the beforementioned innovations in detail we will also illustrate the correlative capabilities of the tomography acquisition software. Here it is possible to align a previously acquired 3D $\mathrm{X}$-ray microscopy (XRM) dataset with the sample under SEM view and precisely target a buried region of interest that may not be noticeable from the surface. For this the multi-resolution workflow that comes with modern $\mathrm{x}$-ray microscopes is especially beneficial. The presented software package allows to first import and align an overview dataset at lower resolution and afterwards add a zoomed-in higher resolution dataset which is then automatically placed at its correct position and inherently aligned.

Using real-world application examples, we will present on how stability and usability of FIB-SEM tomography can be increased. The new features and solutions pave the way to better high-resolution data even of challenging samples. 


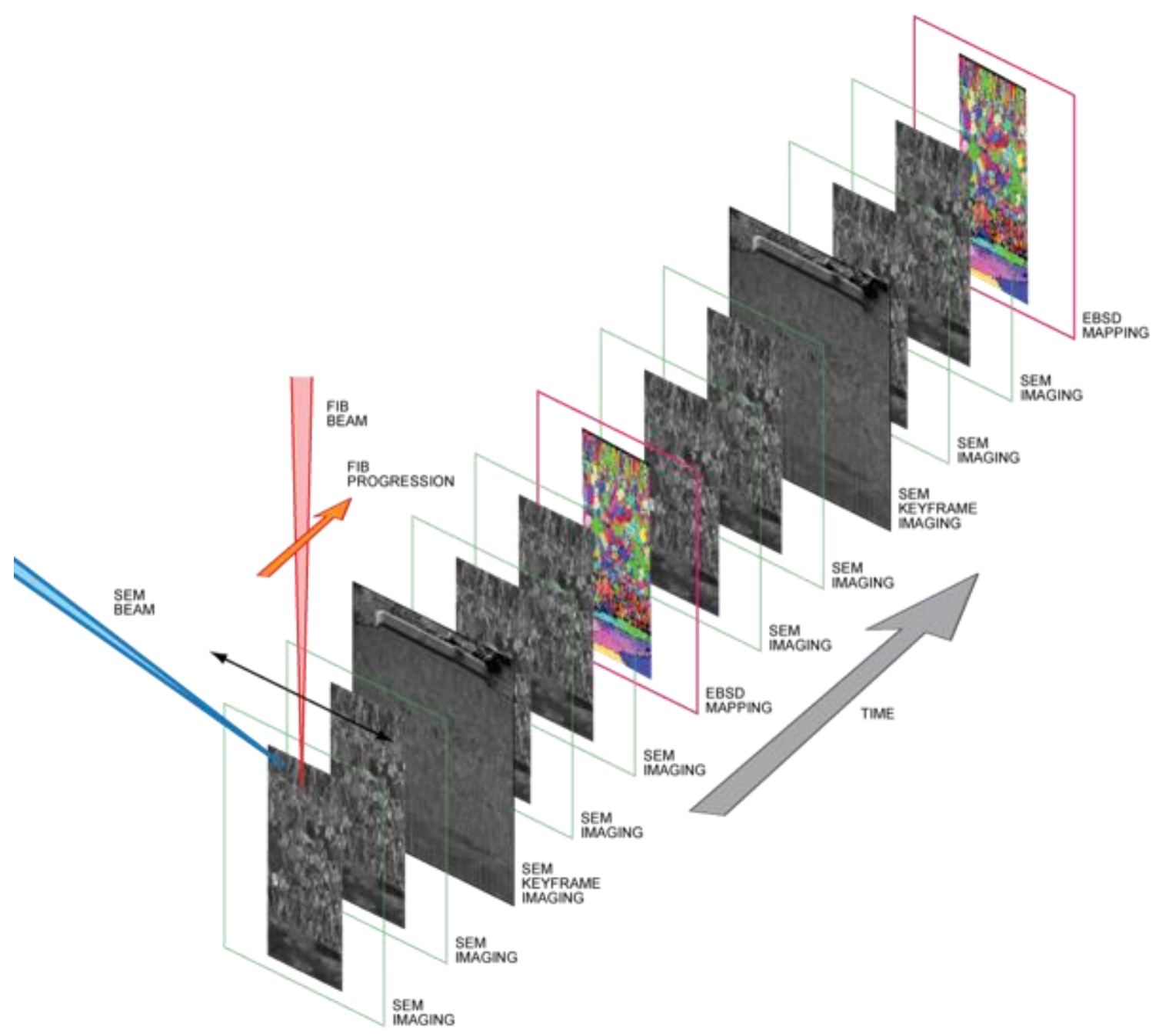

Figure 1. Schematic illustration of a FIB-SEM tomography workflow.

References

[1] https://www.zeiss.com/microscopy/int/products/microscope-software/atlas.html 